\title{
Modulation of target recollection and recollection rejection networks due to retrieval facilitation and interference
}

\author{
Caitlin R. Bowman, ${ }^{1,2,3}$ Shalome L. Sine, ${ }^{1,3}$ and Nancy A. Dennis ${ }^{1}$ \\ ${ }^{1}$ The Department of Psychology, The Pennsylvania State University, University Park, Pennsylvania 16802, USA; ${ }^{2}$ The Department of \\ Psychology, University of Oregon, Eugene, Oregon 97403, USA
}

\begin{abstract}
To better understand neural recollection processing, we induced interference in target recollection by presenting related lures before their respective targets and facilitated recollection rejection of lures by presenting targets before their related lures. Target recollection following interference recruited visual and prefrontal cortices, showing that these regions support recollection when related information has disrupted target representations. Recollection rejection following target presentation recruited angular gyrus, indicating that this region supports recollection rejection when target representations are strong and highly accessible. Thus, recollection networks are sensitive to the accessibility of target representations that are affected by the presentation of related information during retrieval.
\end{abstract}

[Supplemental material is available for this article.]

Memories that involve vivid retrieval of specific information from a prior event are known as recollections (Tulving 1985; Yonelinas 2002; Eichenbaum and Cohen 2008). During recognition, recollection can be used to accept targets based on reinstatement of contextual aspects of an item's original presentation (Yonelinas et al. 1996; Heathcote et al. 2006). Recollection can also be used to reject lures (i.e., recollection rejection) (Brainerd et al. 2003; Lampinen et al. 2004) based on inconsistencies between retrieved details and the currently presented lure. Thus, target recollection and recollection rejection are integral to maintaining memory accuracy and specificity.

Neuroimaging studies have shown that target recollection is typically associated with activation in sensory cortices (Wheeler et al. 2000; Johnson and Rugg 2007), the medial temporal lobes (MTL) (Eldridge et al. 2000; Daselaar 2006), and medial prefrontal and medial parietal regions (Kim 2010). Together, these regions reinstate elements of previous experiences and reconstruct them into episodes (Damasio 1989; Schacter et al. 1998; Rugg et al. 2015). Conversely, recollection rejection has been associated with activation in large portions of lateral prefrontal and lateral parietal cortices that support monitoring and evaluation during retrieval (Lepage et al. 2003; Achim and Lepage 2005; Gallo et al. 2006, 2010; Bowman and Dennis 2016) —-functions that are under high demand when lures resemble targets and recollection rejection is needed to distinguish between similar items. Interestingly, these networks are largely nonoverlapping despite a common recollection response, including a lack of common MTL activation (see Bowman and Dennis 2016 for detailed discussion).

Although the brain networks subserving target recollection and recollection rejection differ, we know that memory judgments for targets and lures can be related. For example, presenting a target immediately before its related distractor can reduce false recognition rates compared with when there are intervening trials between the target and its lure (Brainerd et al. 1995; but see Wallace et al.

\footnotetext{
${ }^{3}$ These authors contributed equally to this work. Corresponding author: nad12@psu.edu Article is online at http://www.learnmem.org/cgi/doi/10.1101/lm.045435.117.
}

2000). Accounts of this effect posit that re-presentation of the target strengthens representations of perceptual details that distinguish between highly similar items and leaves them available in memory when the lure immediately follows. When the lure is presented prior to the target, the number of intervening trials may have little effect on true recognition (Brainerd et al. 1995). This result is somewhat surprising as one might expect lures to cause interference, disrupting or weakening memory for target details. However, it is possible that such lure interference specifically disrupts memory for perceptual details and thus reduces recollection, which was not separated from general recognition without retrieval of details from encoding (i.e., familiarity) in previous studies.

In the present study, we manipulated the order of target-lure presentation in order to generate interference and facilitation during retrieval. Participants made subjective recollection judgments (Tulving 1985) so that we could compare brain activity associated with target recollection occurring before and after lure presentation to identify regions sensitive to interference from lures. We also compared brain activity associated with recollection rejection occurring before and after target presentation to identify regions sensitive to facilitation from targets. Differences in demand caused by target-lure presentation order can tell us more about the roles of individual regions within the broader target recollection and recollection rejection networks defined in a previous analysis of the present data without regard to presentation order (Bowman and Dennis 2016). We expected that recollection responses with high demand due to interference or lack of facilitation would show increased activation in visual regions supporting intensive processing of perceptual details and prefrontal regions supporting memory monitoring of weakened memory traces. We also expected that more difficult recollection decisions would not uniformly increase activation across recollection networks, but rather portions of

(C) 2017 Bowman et al. This article is distributed exclusively by Cold Spring Harbor Laboratory Press for the first 12 months after the full-issue publication date (see http://learnmem.cshlp.org/site/misc/terms.xhtml). After 12 months, it is available under a Creative Commons License (Attribution-NonCommercial 4.0 International), as described at http://creativecommons.org/licenses/by-nc/ $4.0 \%$. 
these networks would instead support recollection responses either unhindered by interference or facilitated by target re-presentation.

Data from 21 participants (12 females, mean age $=22.3 \mathrm{yr}, \mathrm{SD}=3.04 \mathrm{yr}$, range $=18-28 \mathrm{yr}$ ) were included following six exclusions for incomplete data (1), insufficient trials in a condition of interest (4), and movement $>3 \mathrm{~mm}$ within a run (1). Participants were right-handed, native English speakers and screened for contraindications for functional magnetic resonance imaging (fMRI). Participants provided informed consent and received financial compensation for participation. Procedures were approved by Penn State's Institutional Review Board.

Three hundred and sixteen object images were collected from the Bank of Standardized Stimuli database (Brodeur et al. 2010) and an Internet image search. Images were equated for resolution, resized to $\sim 400 \times 400$ pixels, and displayed at a screen resolution of $1024(\mathrm{H}) \times 768$ (V) at $75 \mathrm{~Hz}$. At the viewing distance of $143 \mathrm{~cm}$, the display area was $20^{\circ}(\mathrm{H}) \times$ $16^{\circ}(\mathrm{V})$ with experimental stimuli subtending $5^{\circ}(\mathrm{H}) \times 4^{\circ}(\mathrm{V})$.

Encoding and retrieval were completed in a Siemens Trio 3T scanner with a 12-channel head coil. During encoding, 96 images were each presented for 1500 msec followed by $500 \mathrm{msec}$ additional responding time. Each item was followed by a variable inter-trial fixation $(M=2470$ msec, SD $=1760 \mathrm{msec}$, range: $1000-12,000 \mathrm{msec}$ ). Participants made a size judgment about each item (i.e., is this item bigger or smaller than a shoebox?). Next, participants completed a filler task (Raven's Progressive Matrices) while undergoing a structural scan (MPRAGE: TR: $1650 \mathrm{msec}$, TE: $2.03 \mathrm{msec}, 256-\mathrm{mm}$ field of view (FOV), 160 saggital slices, $1 \mathrm{~mm}$ slice thickness). Approximately $10 \mathrm{~min}$ elapsed between encoding and retrieval. Participants completed four runs of retrieval while undergoing echoplanar functional scans (descending acquisition, TR: 2500 msec, TE: $25 \mathrm{msec}, 240-\mathrm{mm}$ FOV, 42 axial slices, $3 \mathrm{~mm}$ slice thickness). Participants were presented with the target (e.g., a shirt) plus two types of new items from each category: one alternative exemplar of each target (e.g., a different shirt) and one thematically related item (e.g., a sweater). Importantly, only the former (alterative exemplars) were used to define recollection rejection in the present analyses as they maximized these responses (see Fig. 1). Twenty-eight items from novel categories were also included at retrieval but were not the focus of present analyses. Each item was presented for $3000 \mathrm{msec}$ followed by a variable inter-trial interval $(M=2340 \mathrm{msec}, \mathrm{SD}=1440 \mathrm{msec}$, range $=1000-12,000 \mathrm{msec})$. The target was presented first (T1 condition) for half of the categories while the lure was presented first (L1 condition) for the other half. The distance between a target and its lure ranged between 11 and 285 trials $(M=101.52, \mathrm{SD}=76.15){ }^{4}$

${ }^{4}$ Given the large range of intervening trials between related items, we were interested in whether brain activation was affected by this variable. A neuroimaging analysis that sought to identify activity that varied as a function of number of intervening trials (defined both as raw number of trials and the logarithmic distance) revealed no significant regions of activation.
Participants made memory responses within an adapted "Remember-Know-New" paradigm (Bowman and Dennis 2016) designed to identify target recollection and recollection rejection. Participants responded "Remember" when they thought an item was old and they remembered specific details of its prior presentation. Participants responded "Familiar" when they thought an item was old but they did not remember specific details of its prior presentation. "Unfamiliar" responses indicated that they believed an item was new because it did not resemble or bring to mind anything from study. "Different" responses indicated that they believed the item was new because they could recall aspects of studied items that provided evidence that the item was not previously presented. Analyses focused on "remember" responses to targets (target recollection) and "different" responses to lures (recollection rejection) in order to focus on recollection-based responding across targets and lures. Following retrieval, participants completed a questionnaire that asked about their strategies and were debriefed.

Functional data were analyzed with SPM8 (http://www.fil.ion. ucl.ac.uk/spm). Scans were checked for movement and artifacts using a diagnostic function, TSDiffAna (Freiburg Brain Imaging), spatially realigned to correct for motion, coregistered with each individual's high-resolution anatomical image, spatially normalized to a standard stereotaxic space using the Montreal Neurological Institute template, and spatially smoothed using a 6-mm isotropic Gaussian kernel. Trial-related activity was modeled in the General Linear Model with trial onsets convolved with SPM's canonical hemodynamic response function using trial-by-trial reaction time as the trial duration to account for differences in response times across trials of interest (see below). Statistical parametric maps for each participant were identified by applying linear contrasts to the $\beta$ s for the events of interest. The current analysis focused on four regressors: T1 Target Recollection 


\begin{tabular}{|c|c|c|c|c|c|c|c|}
\hline \multirow[b]{2}{*}{ Region } & \multirow[b]{2}{*}{ BA } & \multirow[b]{2}{*}{$H$} & \multicolumn{3}{|c|}{ Peak coordinate-MNI space } & \multirow[b]{2}{*}{$t$} & \multirow[b]{2}{*}{$k$} \\
\hline & & & $x$ & $y$ & $z$ & & \\
\hline \multicolumn{8}{|c|}{ Target Recollection with lure interference (L1 "Remember" hit > T1 "Remember" hit) } \\
\hline Middle frontal gyrus & $6 / 8$ & $\mathrm{R}$ & 36 & 11 & 52 & 5.69 & 38 \\
\hline Inferior parietal cortex & 40 & $\mathrm{R}$ & 42 & -46 & 52 & 4.71 & 81 \\
\hline Lateral occipital cortex & 19 & L & -21 & -94 & 22 & 4.98 & 140 \\
\hline Lateral occipital cortex & 19 & $\mathrm{R}$ & 33 & -91 & 22 & 6.71 & 265 \\
\hline Lingual gyrus & 18 & M & -3 & -73 & 1 & 4.62 & 30 \\
\hline \multicolumn{8}{|c|}{ Target Recollection without lure interference (T1 "Remember" hit > L1 "Remember" hit) } \\
\hline Ventromedial prefrontal cortex & $25 / 32$ & M & -6 & 35 & -8 & 5.29 & 61 \\
\hline \multicolumn{8}{|c|}{ Recollection rejection with target facilitation (T1 "Different" correct rejection >L1 "Different" correct rejection) } \\
\hline Angular gyrus & 40 & $\mathrm{R}$ & 51 & -55 & 37 & 5.92 & 49 \\
\hline \multicolumn{8}{|c|}{ Recollection rejection without target facilitation (L1 "Different" correct rejection > T1 "Different" correct rejection) } \\
\hline Fusiform gyrus & 37 & $\mathrm{~L}$ & -27 & -49 & -17 & 5.37 & 56 \\
\hline Cingulate gyrus & $24 / 32$ & $M$ & 6 & 2 & 40 & 4.35 & 33 \\
\hline
\end{tabular}

Regions showing significant differences in activation based on presentation order for target recollection and recollection rejection. (L1) lure presented before target, (T1) target presented before lure, (BA) Brodmann's Area, $(H)$ hemisphere, $(t) t$-statistic, $(k)$ cluster extent (number of voxels).

("Remember" hits where the target was presented first), L1 Target Recollection ("Remember" hits where the lure was presented first), T1 Recollection rejection ("Different" correct rejections where the target was presented first), and L1 Recollection rejection ("Different" correct rejections where the lure was presented first). Other responses were modeled separately as regressors of no interest.

To determine the effects of lure presentation on target recollection, we contrasted target recollection responses following lure presentation with those occurring before (target recollection with lure interference) and the reverse (target recollection without interference). To determine effects of target presentation on recollection rejection, we contrasted recollection rejection responses following target presentation with those occurring before (recollection rejection with target facilitation) and the reverse (recollection rejection without target facilitation). All contrasts were computed within a whole-brain gray matter mask defined by the Wake Forest University AAL Pickatlas. To determine a cluster threshold corrected for multiple comparisons at $P<0.05,10,000$ Monte Carlo simulations were implemented by 3dClustSim in AFNI (Cox and Hyde 1997) with search space (gray matter), average smoothness in millimeters estimated from residuals $(x, y, z=$ $11.98,11.73,10.1)$, no resampling, and the uncorrected $p$ threshold $(P<0.001)$ as inputs, resulting in a voxel extent of 30 . Full neuroimaging results including peak coordinates are presented in Table 1.

To identify presentation order effects on recollection-based responses, we first computed a 2 (stimulus: target, lure) $\times 2$ (order: $\mathrm{T} 1, \mathrm{~L} 1)$ repeated-measures ANOVA on "Remember" responses to targets and "Different" to responses to lures. There was no main effect of stimulus $\left(F_{(1,20)}=0.01, P>0.9, \eta^{2}=0.001\right)$, but a significant effect of presentation order $\left(F_{(1,20)}=94.62, P<0.001, \eta^{2}=0.83\right)$ with lower recollection rates when the lure was presented first $(M=0.51, \mathrm{SD}=0.14)$ than when the target was presented first $(M$ $=0.71, \mathrm{SD}=0.13)$. The interaction was not significant $\left(F_{(1,20)}=\right.$ $\left.1.51, P>0.2, \eta^{2}=0.07\right)$. We also computed overall d-primes separately for the $\mathrm{T} 1$ and $\mathrm{L} 1$ conditions and found significantly higher accuracy in the T1 $(M=2.11, \mathrm{SD}=0.87)$ compared with the L1 condition $\left(M=1.28, \mathrm{SD}=0.55 ; t_{(20)}=5.73, P<0.001\right)$. Thus, presenting the target before the lure boosted recollection-based responding for both targets and lures compared with presenting the lure first, which also affected overall memory accuracy (see also Supplemental Materials for an analysis of lure responses contingent on the response to the target). These effects are consistent with previous findings showing that priming subjects with the tar- get just before its corresponding lure greatly reduces false recognition (Brainerd et al. 1995), indicating that target presentation facilitates lure rejection. We also found that responses to targets were affected by lure presentation-presenting the lure before the target reduced estimates of target recollection compared with when the target was encountered prior to the lure. Thus, our behavioral results show evidence of both target facilitation and lure interference.

We also tested whether the effects of interference and facilitation were specific to recollection-based responses or instead applied to correct familiarity-based responses as well. We computed a repeated-measures ANOVA on adjusted familiar/unfamiliar rates ${ }^{5}$ (i.e., "Familiar" targets and "Unfamiliar" lures). Results revealed a significant main effect of stimulus $\left(F_{(1,20)}=7.35, P<0.05, \eta^{2}=\right.$ $0.27)$ such that targets were associated with a greater proportion of familiar responses $(M=0.49, \mathrm{SD}=0.19)$ than lures were with unfamiliar responses $(M=0.32, \mathrm{SD}=0.19)$. More importantly, the main effect of order was not significant $\left(F_{(1,20)}=0.16, P>0.6, \eta^{2}\right.$ $=0.008)$ nor was the interaction $\left(F_{(1,20)}=0.36, P>0.56, \eta^{2}=0.02\right)$. Thus, the order of target/lure presentation affected recollectionbased hits and correct rejections but no other correct responses, indicating that recollection signals are particularly sensitive to reinforcement from targets and interference from lures.

Neuroimaging results revealed that target recollection was associated with greater activation in right middle frontal gyrus, right inferior parietal cortex, and bilateral visual cortices for target recollection following lure interference compared with those occurring prior to lure presentation (L1 "remember" hit> T1 "remember" hit) (see Fig. 2A). Thus, these regions support successful target recollection when interfering information has been presented, altering or disrupting target representations or access to target representations. Such activation may represent, in part, retrieval monitoring that accompanies processing of weaker memories (Henson et al. 1999, 2000). Further, recent work has shown that presenting

5Because the rates of "familiar" responses to targets and "unfamiliar" responses to lures are not independent of their respective recollection-based responses, we calculated adjusted rates that correct for this dependence and assess familiarity effects controlling for differences in recollection-based responding. Adjusted familiar hits were calculated as $p$ ("familiar" hit)/(1-p("remember" hit)), and adjusted unfamiliar rejections were calculated as $p$ ("unfamiliar" correct rejection)/[1-p("different" correct rejection)] (Yonelinas and Jacoby 1995; Duarte et al. 2010). These adjusted rates were computed separately for the target first and lure first conditions. 


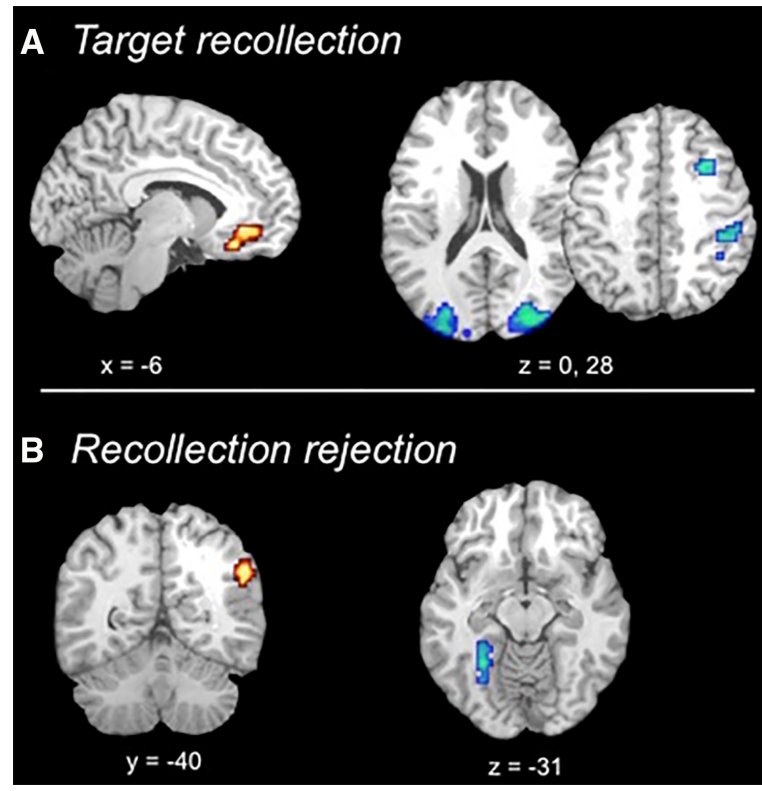

Figure 2. Results of fMRI contrasts. (A) Direct contrasts between lure first and target first conditions for target recollection show increased activation for the target first condition in ventromedial prefrontal cortex (red) and increased activation in visual and frontal regions for the lure first condition (blue). (B) Direct contrasts between lure first and target first conditions for recollection rejection show increased activation for the target first condition in right angular gyrus (red) and increased activation in left fusiform cortex for the lure first condition (blue).

overlapping information in between item repetitions reduces repetition priming compared with repetition without interleaved related information (Zeithamova et al. 2016), which indicates a need to re-parse target information in relationship to other recently experienced, similar information. In the case of L1 target recollections, a related lure is presented between the target's presentation at encoding and that at retrieval. Results thus demonstrate that presenting this related information triggers the need for additional processing to evaluate the target in light of that related information. Alternatively, brain activity associated with target recollection following lure interference may simply reflect strong recollection that was able to withstand lure interference but not residual effects of the interference itself. If this were the case, we would expect strong target recollection following lure presentation to be associated with faster reaction times than those occurring prior to lure presentation (which presumably still include weaker recollections that have not been selected out by interference). Instead, reaction times for $\mathrm{L} 1$ compared with $\mathrm{T} 1$ target recollections were slower $\left(t_{(20)}=3.24, P<0.005\right.$ ) (see Table 2 for means and standard deviations). Therefore, target recollections that survive lure interference nonetheless show traces of processing necessary to negotiate interference, indicating that brain regions associated with these responses likely support effortful target recollection following disruption from lures.

In contrast, activity in the ventromedial prefrontal cortex (VMPFC) was sensitive to lure interference in a different manner: VMPFC showed greater activation for target recollections occurring prior to lure presentations compared with those occurring after. The VMPFC is part of the typical subjective recollection network (Spaniol et al. 2009; Kim 2010), and prior work has shown increased VMPFC activity when retrieved information is congruent with prior knowledge (van Kesteren et al. 2010). The present results further demonstrate that its activation is higher for the target recollection condition with better overall accuracy and faster reaction times compared with disrupted target recollection following lure interference, suggesting that it supports strong recollections based on fluently retrieved details from encoding. Taken together, results show that activation in components of the larger target recollection network are differentially modulated by task demands, with some regions showing increased activation to negotiate interference and other regions showing reduced activation following interference.

For recollection rejection, results revealed increased activation in the right angular gyrus following target facilitation compared with recollection rejection occurring prior to target presentation (see Fig. 2B). The angular gyrus is often active during memory retrieval (Hutchinson et al. 2009; Spaniol et al. 2009), with activation that tracks the vividness of recollection (Vilberg and Rugg 2007), and activation patterns that can be used to decode retrieved content (Kuhl and Chun 2014). This region was also part of the recollection rejection network when presentation order was collapsed (Bowman and Dennis 2016), and its sensitivity to target-lure presentation order suggests that activity in right angular gyrus tracks the strength of target representations that are accessed during recollection rejection. Interestingly, previous work has often found left- rather than right-lateralized memory effects in angular gyrus (for review, see Hutchinson et al. 2009). While the effect reached threshold only on the right in the present study, comparison between activation in right and left angular gyrus revealed no significant difference in the overall pattern of activation across hemispheres (see Supplemental Materials).

Finally, a portion of left fusiform gyrus showed recollection rejection activation that was greater prior to target presentation than following target presentation. Activity in higher-order visual regions during retrieval is typically interpreted as reactivation of visual object information from encoding (Garoff et al. 2005). Thus, when target presentation is relatively remote (i.e., target last presented during encoding phase compared with elsewhere in retrieval), behavioral rates of recollection rejection are reduced, responses are slower, and there is more extensive processing of perceptual information due to its reduced accessibility. Taken together, results demonstrate that activation within the recollection rejection network is sensitive to the strength of target representations, which supports the idea that target representations are accessed as part of this recall-to-reject process. Overall, results demonstrate that presenting related information during retrieval modulates activation within both the target recollection and recollection rejection networks, indicating that portions of these networks are sensitive to the strength of target representations that can be either

Table 2. Behavioral response rates and reaction times

\begin{tabular}{|c|c|c|c|c|}
\hline & \multicolumn{2}{|c|}{ Mean (SD) rates } & \multicolumn{2}{|c|}{$\begin{array}{c}\text { Mean (SD) reaction } \\
\text { times }\end{array}$} \\
\hline & Target first & Lure first & Target first & Lure first \\
\hline \multicolumn{5}{|l|}{ Targets } \\
\hline "Remember" & $0.72(0.14)$ & $0.50(0.17)$ & 1165 (175) & $1261(220)$ \\
\hline "Familiar" & $0.14(0.09)$ & $0.25(0.15)$ & 1974 (332) & $1821(246)$ \\
\hline "Different" & $0.08(0.06)$ & $0.18(0.09)$ & $1837(313)$ & $1675(311)$ \\
\hline "Unfamiliar" & $0.05(0.04)$ & $0.05(0.05)$ & 1555 (374) & 1870 (555) \\
\hline \multicolumn{5}{|l|}{ Lures } \\
\hline "Remember" & $0.06(0.07)$ & $0.14(0.10)$ & 1545 (515) & 1479 (329) \\
\hline "Familiar" & $0.16(0.15)$ & $0.18(0.12)$ & $1882(333)$ & 1969 (409) \\
\hline "Different" & $0.71(0.17)$ & $0.53(0.17)$ & 1459 (208) & 1670 (229) \\
\hline "Unfamiliar" & $0.07(0.05)$ & $0.16(0.08)$ & $1869(662)$ & $1603(282)$ \\
\hline
\end{tabular}

Results showing proportion of each response for each stimulus type separated by presentation order as well as the corresponding reaction time in milliseconds. (SD) standard deviation. 
strengthened by re-presenting the target at retrieval or weakened by presenting other related information.

\section{Acknowledgments}

We would like to thank the Penn State Social, Life, \& Engineering Sciences Imaging Center (SLEIC), 3T MRI Facility. This work was supported by a National Science Foundation grant [BCS1025709] awarded to N.A.D. and dissertation awards granted by the American Psychological Association and Penn State's Research and Graduate Studies Office to C.R.B. Support during manuscript preparation was also provided by National Institute of Aging grant [F32 AG054204-02] awarded to C.R.B.

\section{References}

Achim AM, Lepage M. 2005. Dorsolateral prefrontal cortex involvement in memory post-retrieval monitoring revealed in both item and associative recognition tests. Neuroimage 24: 1113-1121.

Bowman CR, Dennis NA. 2016. The neural basis of recollection rejection: increases in hippocampal-prefrontal connectivity in the absence of a shared recall-to-reject and target recollection network. J Cogn Neurosci 26: $1-16$.

Brainerd C, Reyna V, Kneer R. 1995. False-recognition reversal: when similarity is distinctive. J Mem Lang 34: 157-185.

Brainerd CJ, Reyna VF, Wright R, Mojardin AH. 2003. Recollection rejection: false-memory editing in children and adults. Psychol Rev 110: 762-784.

Brodeur MB, Dionne-Dostie E, Montreuil T, Lepage M. 2010. The bank of standardized stimuli (BOSS), a new set of 480 normative photos of objects to be used as visual stimuli in cognitive research. PLoS One 5: e10773.

Cox RW, Hyde JS. 1997. Software tools for analysis and visualization of fMRI data. NMR Biomed 10: $171-178$.

Damasio AR. 1989. Time-locked multiregional retroactivation: a systems-level proposal for the neural substrates of recall and recognition. Cognition 33: 25-62.

Daselaar SM. 2006. Triple dissociation in the medial temporal lobes: recollection, familiarity, and novelty. J Neurophysiol 96: 1902-1911.

Duarte A, Graham KS, Henson RN. 2010. Age-related changes in neural activity associated with familiarity, recollection and false recognition. Neurobiol Aging 31: 1814-1830.

Eichenbaum H, Cohen NJ. 2008. From conditioning to conscious recollection: memory systems of the brain.

Eldridge LL, Knowlton BJ, Furmanski CS, Bookheimer SY, Engel SA. 2000. Remembering episodes: a selective role for the hippocampus during retrieval. Nat Neurosci 3: 1149-1152.

Gallo DA, Kensinger EA, Schacter DL. 2006. Prefrontal activity and diagnostic monitoring of memory retrieval: FMRI of the criterial recollection task. J Cogn Neurosci 18: 135-148.

Gallo DA, McDonough IM, Scimeca J. 2010. Dissociating source memory decisions in the prefrontal cortex: fMRI of diagnostic and disqualifying monitoring. J Cogn Neurosci 22: 955-969.

Garoff RJ, Slotnick SD, Schacter DL. 2005. The neural origins of specific and general memory: the role of the fusiform cortex. Neuropsychologia 43: 847-859.
Heathcote A, Raymond F, Dunn J. 2006. Recollection and familiarity in recognition memory: evidence from ROC curves. J Mem Lang 55: 495-514.

Henson RNA, Shallice T, Dolan RJ. 1999. Right prefrontal cortex and episodic memory retrieval: a functional MRI test of the monitoring hypothesis. Brain 122: 1367-1381.

Henson RNA, Rugg MD, Shallice T, Dolan RJ. 2000. Confidence in recognition memory for words: dissociating right prefrontal roles in episodic retrieval. J Cogn Neurosci 12: 913-923.

Hutchinson JB, Uncapher MR, Wagner AD. 2009. Posterior parietal cortex and episodic retrieval: convergent and divergent effects of attention and memory. Learn Mem 16: 343-356.

Johnson JD, Rugg MD. 2007. Recollection and the reinstatement of encoding-related cortical activity. Cereb Cortex 17: 2507-2515.

Kim H. 2010. Dissociating the roles of the default-mode, dorsal, and ventral networks in episodic memory retrieval. Neuroimage 50: 1648-1657.

Kuhl BA, Chun MM. 2014. Successful remembering elicits event-specific activity patterns in lateral parietal cortex. J Neurosci 34: 8051-8060.

Lampinen JM, Odegard TN, Neuschatz JS. 2004. Robust recollection rejection in the memory conjunction paradigm. J Exp Psychol Learn Mem Cogn 30: 332-342.

Lepage M, Brodeur M, Bourgouin P. 2003. Prefrontal cortex contribution to associative recognition memory in humans: an event-related functional magnetic resonance imaging study. Neurosci Lett 346: 73-76.

Rugg MD, Johnson JD, Uncapher MR. 2015. Encoding and retrieval in episodic memory: insights from fMRI. In The Wiley handbook on the cognitive neuroscience of memory, pp. 84-107.

Schacter DL, Norman KA, Koutstaal W. 1998. The cognitive neuroscience of constructive memory. Annu Rev Psychol 49: 289-318.

Spaniol J, Davidson PSR, Kim ASN, Han H, Moscovitch M, Grady CL. 2009. Event-related fMRI studies of episodic encoding and retrieval: meta-analyses using activation likelihood estimation. Neuropsychologia 47: 1765-1779.

Tulving E. 1985. Memory and consciousness. Can Psychol 26: 1-12.

van Kesteren MTR, Rijpkema M, Ruiter DJ, Fernandez G, Fernández G. 2010. Retrieval of associative information congruent with prior knowledge is related to increased medial prefrontal activity and connectivity. $J$ Neurosci 30: 15888-15894.

Vilberg KL, Rugg MD. 2007. Dissociation of the neural correlates of recognition memory according to familiarity, recollection, and amount of recollected information. Neuropsychologia 45: 2216-2225.

Wallace WP, Malone CP, Swiergosz MJ, Amberg MD. 2000. On the generality of false recognition reversal. J Mem Lang 43: 561-575.

Wheeler ME, Petersen SE, Buckner RL. 2000. Memory's echo: vivid remembering reactivates sensory-specific cortex. Proc Natl Acad Sci 97: 11125-11129.

Yonelinas A. 2002. The nature of recollection and familiarity: a review of 30 years of research. J Mem Lang 46: 441-517.

Yonelinas AP, Jacoby LL. 1995. The relation between remembering and knowing as bases for recognition: effects of size congruency. J Mem Lang 34: 622-643.

Yonelinas AP, Dobbins I, Szymanski MD, Dhaliwal HS, King L. 1996. Signal-detection, threshold, and dual-process models of recognition memory: ROCs and conscious recollection. Conscious Cogn 5: 418-441.

Zeithamova D, Manthuruthil C, Preston AR. 2016. Repetition suppression in the medial temporal lobe and midbrain is altered by event overlap. Hippocampus 26: 1464-1477.

Received February 28, 2017; accepted in revised form July 28, 2017. 


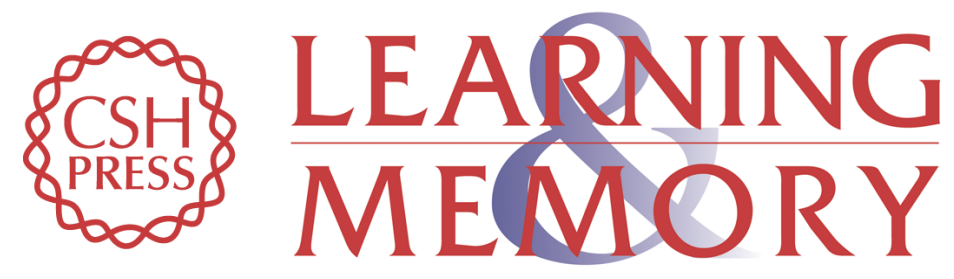

\section{Modulation of target recollection and recollection rejection networks due to retrieval facilitation and interference}

Caitlin R. Bowman, Shalome L. Sine and Nancy A. Dennis

Learn. Mem. 2017, 24:

Access the most recent version at doi:10.1101/Im.045435.117

Supplemental
Material http://learnmem.cshlp.org/content/suppl/2017/10/05/24.11.607.DC1

References This article cites 33 articles, 4 of which can be accessed free at: http://learnmem.cshlp.org/content/24/11/607.full.html\#ref-list-1

Creative This article is distributed exclusively by Cold Spring Harbor Laboratory Press for the Commons first 12 months after the full-issue publication date (see

License http://learnmem.cshlp.org/site/misc/terms.xhtml). After 12 months, it is available under a Creative Commons License (Attribution-NonCommercial 4.0 International), as described at http://creativecommons.org/licenses/by-nc/4.0/.

Email Alerting Receive free email alerts when new articles cite this article - sign up in the box at the Service top right corner of the article or click here. 